\title{
Exploiting SERS sensitivity to monitor DNA aggregation properties
}

\author{
Debora Caprara ${ }^{a}$, Francesca Ripanti a,*, Angela Capocefalo ${ }^{b}$, Marina Ceccarini ${ }^{c}$, \\ Caterina Petrillo ${ }^{\mathrm{d}}$, Paolo Postorino ${ }^{\mathrm{a}}$ \\ a Sapienza University of Rome, P.le Aldo Moro 5, 00185 Rome, Italy \\ ${ }^{\mathrm{b}}$ Istituto dei Sistemi Complessi-CNR c/o Sapienza University of Rome, P.le Aldo Moro 5, 00185 Rome, Italy \\ c National Centre for Rare Diseases, Istituto Superiore di Sanità, Viale Regina Elena 299, 00161 Rome, Italy \\ d Physics and Geology Department, University of Perugia, Via A. Pascoli, 06123 Perugia, Italy
}

\section{A R T I C L E I N F O}

\section{Article history:}

Received 29 September 2020

Received in revised form 19 November 2020

Accepted 5 December 2020

Available online 29 December 2020

\section{Keywords:}

DNA self-assembly

SERS spectroscopy

Melting profile

Silver nanoparticles

\begin{abstract}
A B S T R A C T
In the last decades, DNA has been considered far more than the system carrying the essential genetic instructions. Indeed, because of the remarkable properties of the base-pairing specificity and thermoreversibility of the interactions, DNA plays a central role in the design of innovative architectures at the nanoscale. Here, combining complementary DNA strands with a custom-made solution of silver nanoparticles, we realize plasmonic aggregates to exploit the sensitivity of Surface Enhanced Raman Spectroscopy (SERS) for the identification/detection of the distinctive features of DNA hybridization, both in solution and on dried samples. Moreover, SERS allows monitoring the DNA aggregation process by following the temperature variation of a specific spectroscopic marker associated with the Watson-Crick hydrogen bond formation. This temperature-dependent behavior enables us to precisely reconstruct the melting profile of the selected DNA sequences by spectroscopic measurements only.
\end{abstract}

(c) 2020 Elsevier B.V. All rights reserved.

\section{Introduction}

The outstanding properties of DNA are drawing an increased interest in distinguished applications in many research areas. The rapid progress of cutting edge techniques, such as DNA origami [1,2], contributed to unique scientific advances in structural DNA nanotechnology [3,4]. A large variety of structures with tailored properties have been obtained starting from static 2D or 3D nanostructures [5-10] up to dynamic DNA nanomachines [11-13], feasible platforms for gene analysis, and target detection [14-19]. These achievements result from careful engineering and mastering of the hybridization events, i.e. the formation of a DNA double-stranded complex from two or more complementary single-stranded sequences [20]. Recent advances focus on the implementation of sophisticated and performing systems ensuing from the combination of the self-recognition capability of DNA with the plasmonic properties of metal nanoparticles (NPs). Indeed, the great ability to confine light in a controlled manner at the nanoscale makes metal NPs perfect building blocks for the implementation of smartlydesigned systems with peculiar optical properties for plasmonic and photonic applications [21-25]. On the other hand, the DNA capability to form specific pairing and, thus, well-defined nanoarchitectures, perfectly combines with the necessity of fine-tuning precise NP geometries. This allows the optimization of the plasmonic properties of such systems and ensures the realization of suitable platforms for

\footnotetext{
* Corresponding author.

E-mail address: francesca.ripanti@uniroma1.it (F. Ripanti).
}

surface-enhanced spectroscopies. Among them, Surface Enhanced Raman Spectroscopy (SERS) [26-29] has emerged as the ideal technique for spectroscopic assessment and characterization of hybrid plasmonic systems. Indeed, the nanoantenna effect in metal nanostructures, producing a strong intensification of the electromagnetic field in the vicinity of the metal surface, provides high sensitivity and impressive recognition capability to detect molecules even below micromolar concentrations [30,31].

In this paper, we show how hybridization events between three complementary and purposely programmed single-stranded DNA filaments can be identified via their interaction with silver NPs. The spectroscopic signature of hybridization is thus revealed by the presence of specific spectral markers, appearing in both solution and dried samples. SERS also provides a route to control the hybridization of the complementary DNA strands in solution on varying temperature, thereby enabling the reconstruction of their melting profile. Indeed, as known, the DNA melting is the cooperative unwinding process from a doublestranded DNA complex into two single strands, which generally occurs over a small range of temperature and depends on a variety of factors, such as length of DNA [32], nucleotide sequence composition [33-35], and salt concentration $[33,34,36]$. From the experimental standpoint, different approaches have been adopted to determine the melting temperature of complementary DNA sequences, including UV absorption measurements [37], Differential Scanning Calorimetry studies [38-40], and Circular Dichroism experiments [41]. Additional methods employ specific intercalating fluorophores and quenchers to monitor the DNA double-strand formation/dissociation coupled to the different 
fluorescent intensity profile, registered as a function of temperature [42]. Fluorophore DNA probes are mainly used to obtain the DNA melting curves during the Polymerase Chain Reactions [43] or to reveal the formation of DNA quadruplex [44], molecular beacon [45], and nanoarchitectures [46]. A very recent experimental protocol proposes the simultaneous optical and magnetophoretic monitoring of DNA hybridization using super-paramagnetic and plasmonic colloids [47].

Regarding the possibility to reproduce the melting profile of specific DNA sequences, we propose an innovative approach that exploits the surface selectivity of SERS to control the aggregation/dissociation of complementary sequences as a function of temperature, without using any strand modifications or additional labels. We monitor the SERS response of a specific spectral marker associated with the Watson-Crick base-pairing interactions. The temperature behavior of such a peak, which is well-defined when DNA occurs in the double-stranded configuration and nearly absent once the strands dissociate, allows reproducing the melting profile of the investigated sequences by the analysis of the SERS spectra only. This original result provides an innovative pathway for predicting the DNA melting temperature of complementary and purposely-chosen DNA filaments via a spectroscopic technique that takes advantage of a simple experimental protocol for sample preparation and data collection.

\section{Experimental methods}

\subsection{DNA sequences}

DNA sequences are purchased from Integrated DNA Technologies with HPLC purification. In our experiment, we use three different DNA strands whose composition has been chosen in line with the seminal work of J. J. Storhoff et al. [48], and to whom we refer as chain-A, chain-B, and bridge, respectively. Chain-A and chain-B are 12-base oligonucleotides, whereas the bridge is a 24-base oligomer, complementary to both previous sequences, which acts as a linker for the plasmonic aggregate formation. The specific sequences are:

$$
\begin{aligned}
& \text { chain - A : 5' - CGCATTCAGGAT - 3', } \\
& \text { chain - B: 5' - TCTCAACTCGTA - 3', }
\end{aligned}
$$$$
\text { bridge : } 5^{\prime} \text { - TACGAGTTGAGAATCCTGAATGCG - 3'. }
$$

The lyophilized DNA samples are initially reconstituted in a DNAsefree $0.15 \mathrm{M} \mathrm{NaCl}$ solution at a concentration of $300 \mu \mathrm{M}$ each.

DNA double-stranded complexes ( $\mathrm{A}+\mathrm{B}+$ bridge) are assembled by mixing equimolar quantities of the three single-stranded components to a final concentration of $100 \mu \mathrm{M}$. The mixture is heated up to $75^{\circ} \mathrm{C}$, a temperature value that guarantees the breaking of hydrogen bonds between complementary bases, thus ensuring each filament occurring in the single-stranded configuration, thus leading to the spontaneous formation of the duplexes.

\subsection{Hydroxylamine-reduced silver NPS}

Borrowing from the synthesis protocol proposed by $\mathrm{N}$. Leopold in Ref. [49] and from the seminal works of S.E.J. Bell [50,51], we provide for the realization of a silver NP (Ag-NP) colloidal solution reduced with hydroxylamine. Such a method boasts of a standard reduction reaction that can take place at room temperature and allows for the elimination of the residual oxidation products. Moreover, it enables the realization of large NP aggregates and does not produce interference bands in crucial regions of the Raman spectrum [52].

Firstly, $5 \mathrm{~mL}$ of $0.1 \mathrm{M} \mathrm{NaOH}$ solution are mixed with $5 \mathrm{~mL}$ of aqueous $6 \mathrm{mM}$ hydroxylamine hydrochloride. Parallely, $90 \mathrm{~mL}$ of DNAse-free water are added to $17 \mathrm{mg}$ of powdered $\mathrm{AgNO}_{3}$ to a final $0.1 \mathrm{mM}$ concentration. The two solutions are then mixed up and stirred for about $20 \mathrm{~min}$ at room temperature to allow the spontaneous formation of the colloids [49,51]. The characterization of the silver colloids is reported in Section S2 of the Supplementary data.

\subsection{Raman and SERS measurements}

For Raman and SERS measurements, a Horiba HR-Evolution microspectrometer in backscattering geometry, equipped with a He-Ne laser, $\lambda=632.8 \mathrm{~nm}$, and $30 \mathrm{~mW}$ output power $(\sim 10 \mathrm{~mW}$ at the sample surface) has been used. The detector is a Peltier-cooled Charge-Coupled Device (CCD), and the resolution is better than $3 \mathrm{~cm}^{-1}$ thanks to a 600 grooves/mm grating with $800 \mathrm{~mm}$ focal length. The spectrometer is coupled with a confocal microscope supplied with a set of interchangeable objectives with long working distances and different magnifications. $100 \times($ N.A. $=0.9$ ) and $10 \times($ N.A. $=0.4$ ) Olympus objective lenses are used for the acquisitions on dried and solute samples, respectively. Further details on the experimental apparatus can be found in Ref. [53].

Raman characterization is performed on dried samples by separately dropcasting small volumes of the three different single-chain DNA stock solutions and the hybridized A + B + bridge sample on a planar gold leaf substrate. We then let the solutions dry at room temperature.

In the case of SERS measurements, we performed:

i. measurements in solution - to characterize the SERS fingerprint of the three single DNA chains and of the hybridized system ( $A+B+$ bridge), as well as for the reconstruction of the melting profile of the complementary sequences;

ii. measurements on dried samples - to assess if the spectral information obtained on solute samples still remain after the drying process.

For measurements described in point (i), we initially prepared three diluted solutions of the three single DNA sequences at $100 \mu \mathrm{M}$, and we heated up the samples to $60{ }^{\circ} \mathrm{C}$ for a minute to prevent and remove undesired bindings among the sequences, thus guaranteeing the almost-complete removal of intra-strand bindings (see Fig. S2 of the Supplementary data to clarify the value of such selected temperature). We then diluted 10 times the DNA solution (final concentration of 10 $\mu \mathrm{M}$ ) adding hydroxylamine-reduced colloidal solution and $0.01 \mathrm{M}$ of $\mathrm{MgSO}_{4}$. In the case of the aggregate system, we mixed the three strands at a $100 \mu \mathrm{M}$ of $\mathrm{A}+\mathrm{B}+$ bridge concentration, and we annealed the mixture as previously described. Once the system has hybridized, we mixed that solution with the Ag-NPs following the same preparation procedure used for the single chains, reaching a final concentration of $10 \mu \mathrm{M}$.

$\mathrm{MgSO}_{4}$ salt is used for facilitating the NP aggregation and ensuring an electrostatic interaction of DNA with the metal surface. Indeed, despite the higher stability of the chemically prepared silver colloids, a partial aggregation is necessary for them to become SERS-active and to create the hot-spots necessary for a high signal enhancement. This aggregation leads to the coupling between the localized plasmonic modes of the single NPs, resulting in the frequency shifts of the localized surface plasmon resonance (LSPR) of the aggregated system towards higher wavelengths [54,55], implying the use of a red laser.

SERS spectra were collected on $200 \mu$ L of DNA-NP solutions, loaded into separate polypropylene microplate wells (Greiner Bio-One). Conversely, to reconstruct the melting profile of the three complementary sequences, we loaded a quartz cuvette with $200 \mu \mathrm{L}$ of the Ag-NP solution mixed with the $A+B+$ bridge aggregate system. The simulated melting profile of the $A+B+$ bridge sample at $10 \mu \mathrm{M}$ is shown in Fig. S3 of the Supplementary data, including in the calculation the presence of the additional $\mathrm{MgSO}_{4}$ at $1 \mathrm{mM}$. We note that the value of the melting temperature is $\mathrm{T}_{\mathrm{M}} \sim 53^{\circ} \mathrm{C}$ and does not differ enough from the one calculated for the batch sample reported in Fig. S1. Measurements were thus collected in a temperature range between $\mathrm{T}_{\mathrm{i}} \sim 20^{\circ} \mathrm{C}$ (when all the duplexes are formed) to $\mathrm{T}_{\mathrm{f}} \sim 72^{\circ} \mathrm{C}$ ( single-stranded configuration).

For measurements on dried samples pointed out in (ii), single drops $(\sim 2 \mu \mathrm{L})$ of the same samples (single chains and aggregate $\mathrm{A}+\mathrm{B}+$ bridge 
system) measured in solution and described in point (i) are dropcasted on a gold leaf substrate and let them dry at room temperature.

\section{Results and discussion}

\subsection{Raman characterization}

In Fig. 1, we show the Raman spectra of the three single chains (chain- $A$, chain-B, and bridge) and of the aggregate system (hybridized system: $A+B+$ bridge), which is formed after the annealing process, as described in the Experimental methods.

The measurements on dried samples provide to obtain Raman spectra of the DNA sequences with a signal-to-noise ratio much higher than data collected in water solution. Indeed, as reported in previous investigations, large amounts of sample are typically required in solutions $[56,57]$ due to the low-Raman cross-section of DNA; conversely, in our case, we work with well-purified sequences that allow obtaining spectral information even on dried samples. All spectra are characterized by an intense band at $\sim 789 \mathrm{~cm}^{-1}$ ascribed to the vibration of both the cytosine base and the O-P-O phosphate backbone, which, since its invariance to the melting, has already been used as a good reference peak for normalization $[39,58]$. The high quality of the spectra allows for the precise identification of the DNA spectral fingerprints associated with the four different nitrogen bases, as highlighted by the vertical arrows in Fig. 1. Specifically, the peaks at $729 \mathrm{~cm}^{-1}$ and $670 \mathrm{~cm}^{-1}$ refer to the ring breathing modes of adenine $(A)$ and guanine $(G)$ respectively, while the contribution at $594 \mathrm{~cm}^{-1}$ is relative to cytosine (C), and the one at $1370 \mathrm{~cm}^{-1}$ to thymine $(\mathrm{T})$.

The presence of the spectroscopic marker of hybridization at $\sim 1680 \mathrm{~cm}^{-1}$, relative to the Watson-Crick complementary pairing via hydrogen bonds $[39,57]$ and evident in the spectrum of the aggregate system ( $\mathrm{A}+\mathrm{B}+$ bridge), allows to finely distinguish the single- and double-stranded DNA species (see the vertical dashed line in Fig. 1). We notice that, although the occurrence of the hybridization spectroscopic marker is quite evident in the aggregate spectrum, weak shoulders can also be observed in the spectra of the single chains, particularly in the bridge. Since spectra are collected from dried samples, the effective pairing process is not precisely controlled, and spurious hydrogen bonds between complementary bases, belonging to the same or distinct strands (i.e. self- or inter-chain folding), can mainly occur for the bridge, which is the longest DNA sequence.

\subsection{SERS measurements on solution samples}

SERS spectra of both single chains and aggregate system are shown in Fig. 2. According to the Raman spectra of Fig. 1, the spectroscopic features associated with the principal vibrational modes of the nucleotides

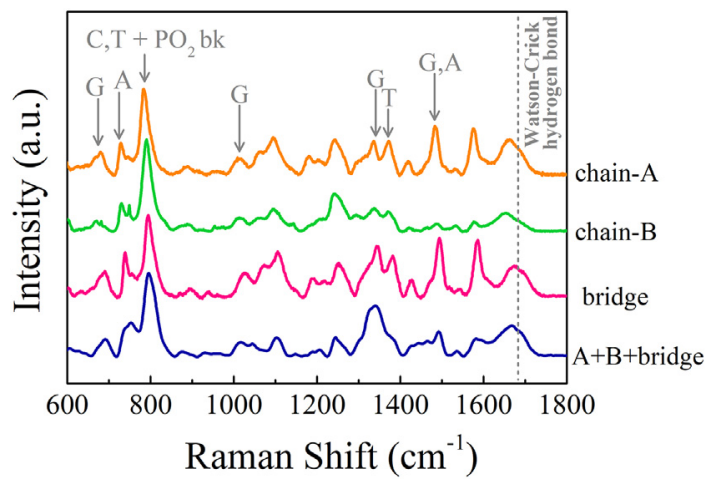

Fig. 1. Raman spectra of the three complementary chains, chain-A (orange), chain-B (green) and bridge (pink), and the aggregate system ( $\mathrm{A}+\mathrm{B}+$ bridge, blue). All spectra are collected on dried samples. The arrows indicate the principal vibrational modes relative to the single bases (A: adenine, C: cytosine, G: guanine, T: thymine). The dashed line highlights the spectroscopic marker of hybridization at $\sim 1680 \mathrm{~cm}^{-1}$.

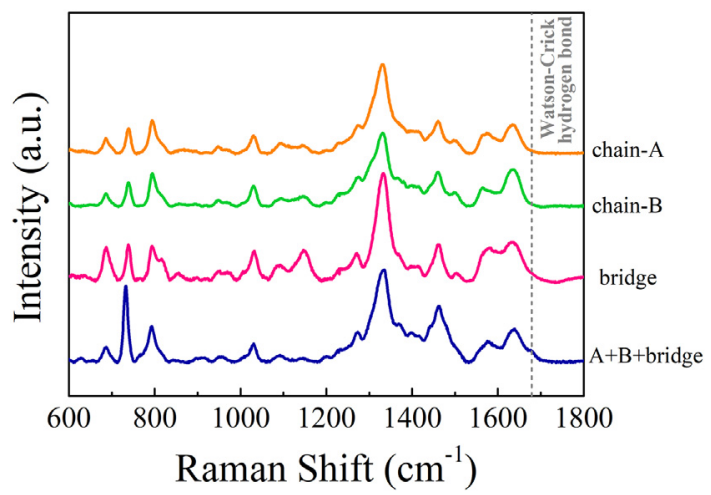

Fig. 2. SERS spectra of the three complementary chains, chain-A (orange), chain-B (green), and bridge (pink), and of the combined system ( $A+B+$ bridge, blue). The main differences between single- and double-stranded chains are evidenced.

are evident in all the SERS spectra. Some of them appear enhanced and red-shifted due to the SERS effect promoted by the metal NPs [59].

The first eye-catching difference in the spectrum of the aggregate system with respect to single chains is the higher intensity of the peak at $\sim 730 \mathrm{~cm}^{-1}$ (adenine in-plane ring vibration). This enhancement of the ring breathing mode suggests, according to previous literature, that the adenine base aligns perpendicularly to the metal surface, even in the double helix configuration [60]. A close inspection of Fig. 2 points out a clean hybridization process without spurious pairing as the hydrogen bond peak is observed for the aggregate system only. This provides for a high SERS sensitivity to hybridization events, and SERS, in turn, can be exploited to control the correct pairing of the three DNA complementary strands. Such a high sensitivity allowed us to monitor the temperature dependence of the system aggregation and to check the reversibility of the hybridization process. In particular, we aim at controlling the pairing/unpairing processes below and above the DNA melting temperature of the studied strands, which falls at $\mathrm{T}_{\mathrm{M}} \sim 53^{\circ} \mathrm{C}$, as reported in Fig. S3 of the Supplementary data.

To this purpose, we started from a solution of Ag-NPs and DNA hybridized system $(A+B+$ bridge $)$ at room temperature $\left(T_{i}<T_{M}\right)$. Following, we raise the temperature up to $\mathrm{T}_{\mathrm{f}} \sim 72^{\circ} \mathrm{C}\left(\mathrm{T}_{\mathrm{f}}>\mathrm{T}_{\mathrm{M}}\right)$, ensuring the unwinding of double-stranded complexes. The unpairing of the double strands in favor of single chains is actually expected on increasing temperature from $T_{i}$. The resulting spectra are shown in Fig. 3 as a function of temperature. A clear temperature trend emerges from the collected measurements: the spectroscopic marker of hybridization at $\sim 1680 \mathrm{~cm}^{-1}$, which is well-visible in the low-temperature region, starts to disappear around $50^{\circ} \mathrm{C}$, namely at a temperature closer to the melting temperature of the three strands. The peak almost disappears above $\mathrm{T}_{\mathrm{M}}$, although a small contribution is still observed in the spectrum collected

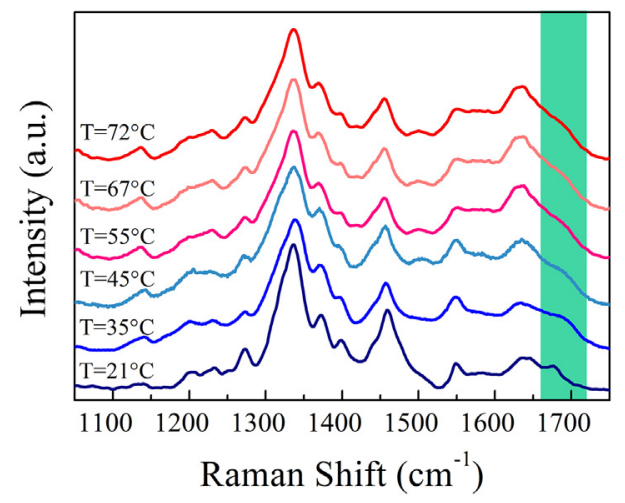

Fig. 3. SERS spectra of the three hybridized complementary strands ( $A+B+$ bridge) for selected temperature values, in the enlarged $1050-1750 \mathrm{~cm}^{-1}$ spectral range. The green box highlights the spectroscopic marker of hybridization at $\sim 1680 \mathrm{~cm}^{-1}$. 
at $T_{f}$, where, in principle, only single strands should exist. A possible explanation of this behavior is suggested by the complexity of the system under investigation: indeed, the DNA strands trapped inside the NP aggregates can not freely diffuse and completely dissociate in solution, resulting in a small fraction of double-stranded species still present at high-temperature.

The reversibility of the process is found by repeating the same measurements on slowly cooling down the solution to room temperature. An accurate fit of the experimental data in the $[1500-1750] \mathrm{cm}^{-1} \mathrm{spec}-$ tral range with Gaussian functions was performed to analyze the intensity variation of the hybridization spectroscopic marker at $\sim 1680 \mathrm{~cm}^{-1}$, which allows to quantitatively describe the temperature behavior of the system. Indeed, as shown in Fig. 4, we evaluated the peak area on increasing (blue points) and on decreasing (red points) the temperature. Further details regarding the fitting procedure can be found in Section S3 of the Supplementary data. In Fig. 4, we also show the theoretical melting profile (gray line), extrapolated from NUPACK oligo simulator, an online platform that provides to compute the melting temperature of complementary DNA sequences at the precise strand and salt concentrations [61]. A very good agreement is found between the theoretical curve and the experimental data collected on increasing the temperature (blue points), while, on decreasing the temperature (red points), a sort of hysteresis behavior is observed, suggesting that steric hindrance effects could influence the kinetics of the melting process on cooling, resulting in different amounts of hybridized sequences.

In summary, a clear and quite evident effect on varying temperature is observed, strongly suggesting that SERS spectroscopy can be efficiently applied to monitor the melting behavior of complementary DNA chains.

\subsection{SERS measurements on dried samples}

To evaluate if hybridization between complementary sequences remains stable during the drying process and if the relative spectroscopic signature can be observed even in dried samples, we performed SERS measurements of the single chains and aggregate system after deposition and drying of the respective solutions. The obtained spectra are shown in Fig. 5, where the DNA peaks are clearly observable, but some contributions ascribed to the hydroxylamine, the molecule used for the Ag-NP synthesis (see Experimental methods), can also be identified. The hydroxylamine spectrum is shown in the inset of Fig. 5 for comparison (see Fig. S5 of the Supplementary data for the spectrum in the extended range and the assignment of the principal bands). We note that the peaks corresponding to hydroxylamine, visible in the spectra of Fig. 5, are not observed in the solution samples plotted in Fig. 2.

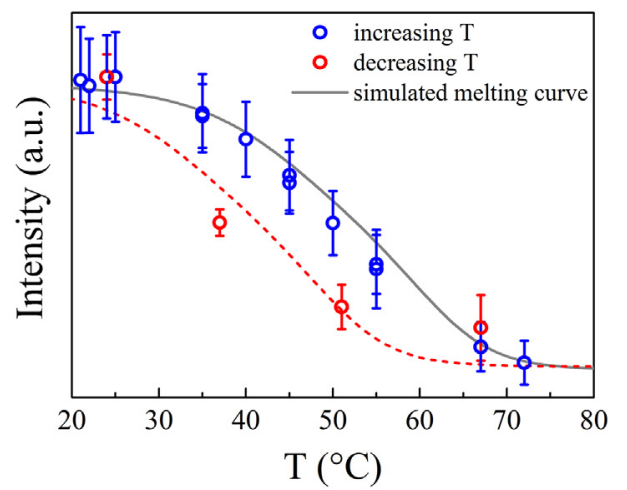

Fig. 4. Area of the peak at $1680 \mathrm{~cm}^{-1}$ on increasing (blue dots) and on lowering (red dots) the temperature. The theoretical melting curve of the system is reported for comparison (gray line). Error bars are estimated as the area of the tails that intersect the overall area of the considered peak in the fitting procedure (see Section S3 of the Supplementary data). The dashed red line represents a guide to the eye for the experimental data obtained on lowering the temperature. Overlapping dots are the result of repeated measurements.

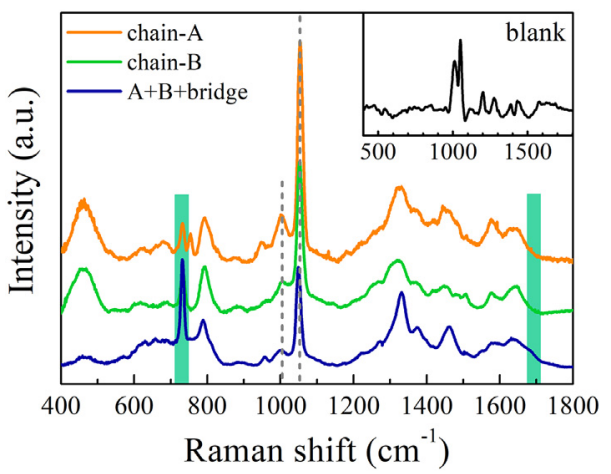

Fig. 5. SERS spectra of the single chains and the aggregate system in dried form. The green boxes indicate the spectral differences between single- and double-stranded conformations. The contributions relative to the hydroxylamine molecule, coming from the preparation protocol of Ag-NPs, are highlighted by dashed lines. The entire hydroxylamine spectrum is reported in the inset.

This evidence can be attributed to the drying process, during which a closer packing of the NPs and the hydroxylamine molecules can occur.

However, the presence of the spectroscopic marker associated with the hybridization at $1680 \mathrm{~cm}^{-1}$ is still visible in the spectrum of the aggregate sample, and it is not affected by the presence of hydroxylamine. This proves that the double-stranded complexes formed in solution at low temperature remain paired during the drying process.

Moreover, as in the SERS spectra of solution samples, reported in Fig. 2, the adenine peak at $730 \mathrm{~cm}^{-1}$ results more intense than the contribution registered in the single chains, suggesting an intrinsic modification of the system due to the geometrical packing of the chains on the metal surface: the double strands take on a more packed geometry on the metal surface with respect to the single filaments.

All these effects, observed in the dried samples, are consistent with those obtained in solution samples, proving the efficacy of SERS spectroscopy for discriminating the hybridization occurrence both in solution and on dried samples.

\section{Conclusions}

Over the last years, great attention has been focused on the implementation of different methodologies for the production of plasmonic nanoarchitectures with tailored and controllable properties. Here, SERS spectroscopy is exploited and turns out to be a powerful technique to characterize the spectroscopic signal of complementary DNA sequences electrostatically interacting with a solution of silver NPs. The obtained results arise from a fruitful combination of an experimental procedure for the rapid, cost-effective synthesis of metal NPs and a simple data acquisition. The high sensitivity of the technique is exploited to detect the conformational changes of DNA, from the single- to the double-stranded configuration, proven by the presence of the specific peak ascribed to the formation of hydrogen bonds between complementary bases. The fingerprint of hybridization is revealed in both solution and dried samples. Moreover, since the DNA pairing is a reversible process, we controlled the temperature dependence of the aggregation/ dissociation of our complementary sequences without making use of any strand modifications or additional labels.

Analyzing the intensity of the spectroscopic feature associated with the occurring hybridization as a function of temperature, we are able to reproduce the melting profile of the DNA strands. The temperature behavior of the system, resulting from a different response in the collected SERS spectra, reveals a clear change in the DNA conformation switching from a completely bound to an almost disentangled structure.

In conclusion, the ability to monitor and control the DNA melting process constitutes a challenging effort for the outstanding implications aiming at deciphering molecular details of replication and transcription [62,63], gene expression [64,65], DNA mutations [66,67], and, more 
generally, genome organization [68]. Further improvements and attempts are therefore well worthwhile for the broad perspectives and possible applications in several research fields, addressing to the design and development of more complex nanostructures with appealing properties for sensing and nanotechnological devices.

\section{CRediT authorship contribution statement}

Debora Caprara: Investigation, Formal Analysis, Writing Original Draft

Francesca Ripanti: Conceptualization, Investigation, Formal Analysis, Validation, Writing - Review \& Editing

Angela Capocefalo: Investigation, Formal Analysis, Writing - Review \& Editing

Marina Ceccarini: Resources, Writing - Review \& Editing

Caterina Petrillo: Validation, Funding acquisition, Writing - Review \&

Editing, Supervision

Paolo Postorino: Conceptualization, Writing - Original Draft, Writing - Review \& Editing, Supervision

\section{Declaration of competing interest}

There are no conflicts to declare.

\section{Acknowledgements}

The authors would like to thank Dr. Francesco Mura for the technical support in SEM imaging, and Prof. Federico Bordi and Dr. Simona Sennato for providing the access to the DLS instrumentation. We also thank Prof. Alessandro Nucara for useful discussions.

\section{Appendix A. Supplementary data}

Supplementary data to this article can be found online at https://doi. org/10.1016/j.ijbiomac.2020.12.039.

\section{References}

[1] P.W. Rothemund, Folding DNA to create nanoscale shapes and patterns, Nature 440 (7082) (2006) 297.

[2] A. Kuzuya, M. Komiyama, DNA origami: fold, stick, and beyond, Nanoscale 2 (3) (2010) 309-321.

[3] N.C. Seeman, DNA in a material world, Nature 421 (6921) (2003) 427.

[4] N.C. Seeman, H.F. Sleiman, DNA nanotechnology, Nat. Rev. Mater. 3 (2017) 17068.

[5] V.V. Thacker, L.O. Herrmann, D.O. Sigle, T. Zhang, T. Liedl, J.J. Baumberg, U.F. Keyser, DNA origami based assembly of gold nanoparticle dimers for surface-enhanced Raman scattering, Nat. Commun. 5 (2014) 3448.

[6] S.M. Douglas, H. Dietz, T. Liedl, B. Högberg, F. Graf, W.M. Shih, Self-assembly of DNA into nanoscale three-dimensional shapes, Nature 459 (7245) (2009) 414.

[7] H. Dietz, S.M. Douglas, W.M. Shih, Folding DNA into twisted and curved nanoscale shapes, Science 325 (5941) (2009) 725-730.

[8] T. Liedl, B. Högberg, J. Tytell, D.E. Ingber, W.M. Shih, Self-assembly of threedimensional prestressed tensegrity structures from DNA, Nat. Nanotechnol. 5 (7) (2010) 520 .

[9] D. Han, S. Pal, J. Nangreave, Z. Deng, Y. Liu, H. Yan, DNA origami with complex curvatures in three-dimensional space, Science 332 (6027) (2011) 342-346.

[10] R. Iinuma, Y. Ke, R. Jungmann, T. Schlichthaerle, J.B. Woehrstein, P. Yin, Polyhedra self-assembled from DNA tripods and characterized with 3D DNA-PAINT, Science 1250944 (2014)

[11] Y. Ke, L.L. Ong, W.M. Shih, P. Yin, Three-dimensional structures self-assembled from DNA bricks, Science 338 (6111) (2012) 1177-1183.

[12] B. Wei, M. Dai, P. Yin, Complex shapes self-assembled from single-stranded DNA tiles, Nature 485 (7400) (2012) 623.

[13] D. Han, S. Pal, Y. Yang, S. Jiang, J. Nangreave, Y. Liu, H. Yan, DNA gridiron nanostructures based on four-arm junctions, Science 339 (6126) (2013) 1412-1415.

[14] O. Neumann, D. Zhang, F. Tam, S. Lal, P. Wittung-Stafshede, N.J. Halas, Direct optical detection of aptamer conformational changes induced by target molecules, Anal. Chem. 81 (24) (2009) 10002-10006.

[15] L. He, E. Lamont, B. Veeregowda, S. Sreevatsan, C.L. Haynes, F. Diez-Gonzalez, T.P. Labuza, Aptamer-based surface-enhanced Raman scattering detection of ricin in liquid foods, Chem. Sci. 2 (8) (2011) 1579-1582.
[16] J. Prinz, C. Heck, L. Ellerik, V. Merk, I. Bald, DNA origami based Au-Ag-core-shell nanoparticle dimers with single-molecule SERS sensitivity, Nanoscale 8 (10) (2016) 5612-5620.

[17] J.-W. Keum, M. Kim, J.-M. Park, C. Yoo, N. Huh, S.C. Park, DNA-directed self-assembly of three-dimensional plasmonic nanostructures for detection by surface-enhanced Raman scattering (SERS), Sensing and Bio-sensing Research 1 (2014) 21-25.

[18] X. Yu, Z.-L. Zhang, S.-Y. Zheng, Highly sensitive DNA detection using cascade amplification strategy based on hybridization chain reaction and enzyme-induced metallization, Biosens. Bioelectron. 66 (2015) 520-526.

[19] Z. Zhou, G. Luo, V. Wulf, I. Willner, Application of DNA machineries for the barcode patterned detection of genes or proteins, Anal. Chem. 90 (11) (2018) 6468-6476.

[20] N.H. Kim, S.J. Lee, M. Moskovits, Reversible tuning of SERS hot spots with aptamers, Adv. Mater. 23 (36) (2011) 4152-4156.

[21] R.J. Macfarlane, B. Lee, M.R. Jones, N. Harris, G.C. Schatz, C.A. Mirkin, Nanoparticle superlattice engineering with DNA, Science 334 (6053) (2011) 204-208.

[22] Y.-C. Yeh, B. Creran, V.M. Rotello, Gold nanoparticles: preparation, properties, and applications in bionanotechnology, Nanoscale 4 (6) (2012) 1871-1880.

[23] S.Y. Park, A.K. Lytton-Jean, B. Lee, S. Weigand, G.C. Schatz, C.A. Mirkin, DNAprogrammable nanoparticle crystallization, Nature 451 (7178) (2008) 553.

[24] E. Auyeung, T.I. Li, A.J. Senesi, A.L. Schmucker, B.C. Pals, M.O. de La Cruz, C.A. Mirkin, DNA-mediated nanoparticle crystallization into Wulff polyhedra, Nature 505 (7481) (2014) 73.

[25] H.T. Ngo, H.-N. Wang, A.M. Fales, T. Vo-Dinh, Plasmonic SERS biosensing nanochips for DNA detection, Anal. Bioanal. Chem. 408 (7) (2016) 1773-1781.

[26] M. Moskovits, Surface-enhanced spectroscopy, Rev. Mod. Phys. 57 (3) (1985) 783.

[27] P.L. Stiles, J.A. Dieringer, N.C. Shah, R.P. Van Duyne, Surface-enhanced Raman spectroscopy, Annu. Rev. of Anal. Chem. 1 (1) (2008) 601-626.

[28] J. Langer, D. Jimenez de Aberasturi, J. Aizpurua, R.A. Alvarez-Puebla, B. Auguié, J.J Baumberg, G.C. Bazan, S.E. Bell, A. Boisen, A.G. Brolo, et al., Present and future of surface-enhanced Raman scattering, ACS Nano 14 (1) (2019) 28-117.

[29] F. Domenici, C. Fasolato, E. Mazzi, L. De Angelis, F. Brasili, F. Mura, P. Postorino, F. Bordi, Engineering microscale two-dimensional gold nanoparticle cluster arrays for advanced Raman sensing: an AFM study, Colloids Surf. A Physicochem. Eng. Asp. 498 (2016) 168-175.

[30] B. Gu, C. Ruan, W. Wang, Perchlorate detection at nanomolar concentrations by surface-enhanced Raman scattering, Appl. Spectrosc. 63 (1) (2009) 98-102.

[31] A. Capocefalo, D. Mammucari, F. Brasili, C. Fasolato, F. Bordi, P. Postorino, F. Domenici, Exploring the potentiality of a SERS-active pH nano-biosensor, Frontiers in Chemistry 7 (2019) 413.

[32] D. Pörschke, M. Eigen, Co-operative non-enzymatic base recognition III. Kinetics of the helix-coil transition of the oligoribouridylic. oligoriboadenylic acid system and of oligoriboadenylic acid alone at acidic pH, J. Mol. Biol. 62 (2) (1971) 361-381.

[33] K.J. Breslauer, R. Frank, H. Blöcker, L.A. Marky, Predicting DNA duplex stability from the base sequence, Proc. Natl. Acad. Sci. U. S. A. 83 (11) (1986) 3746-3750.

[34] S.G. Delcourt, R. Blake, Stacking energies in DNA, J. Biol. Chem. 266 (23) (1991) 15160-15169.

[35] I. Lafontaine, R. Lavery, Optimization of nucleic acid sequences, Biophys. J. 79 (2) (2000) 680-685.

[36] J. Marmur, P. Doty, Determination of the base composition of deoxyribonucleic acid from its thermal denaturation temperature, J. Mol. Biol. 5 (1) (1962) 109-118.

[37] R. Thomas, The denaturation of DNA, Gene 135 (1-2) (1993) 77-79.

[38] Y.-W. Park, K.J. Breslauer, A spectroscopic and calorimetric study of the melting behaviors of a "bent" and a "normal" DNA duplex:[d (GA4T4C)] 2 versus [d (GT4A4C)] 2, Proc. Natl. Acad. Sci. U. S. A. 88 (4) (1991) 1551-1555.

[39] J.G. Duguid, V.A. Bloomfield, J.M. Benevides, G.J. Thomas Jr., DNA melting investigated by differential scanning calorimetry and Raman spectroscopy, Biophys. J. 71 (6) (1996) 3350-3360.

[40] C.H. Spink, Differential scanning calorimetry, Methods Cell Biol. 84 (2008) 115-141.

[41] J. Kypr, I. Kejnovská, D. Renčiuk, M. Vorlčková, Circular dichroism and conformational polymorphism of DNA, Nucleic Acids Res. 37 (6) (2009) 1713-1725.

[42] Y. You, A.V. Tataurov, R. Owczarzy, Measuring thermodynamic details of DNA hybridization using fluorescence, Biopolymers 95 (7) (2011) 472-486.

[43] S. Giglio, P.T. Monis, C.P. Saint, Demonstration of preferential binding of SYBR Green I to specific DNA fragments in real-time multiplex PCR, Nucleic Acids Res. 31 (22) (2003) e136.

[44] A. De Cian, L. Guittat, M. Kaiser, B. Saccà, S. Amrane, A. Bourdoncle, P. Alberti, M.-P. Teulade-Fichou, L. Lacroix, J.-L. Mergny, Fluorescence-based melting assays for studying quadruplex ligands, Methods 42 (2) (2007) 183-195.

[45] A. Tsourkas, M.A. Behlke, S.D. Rose, G. Bao, Hybridization kinetics and thermodynamics of molecular beacons, Nucleic Acids Res. 31 (4) (2003) 1319-1330.

[46] B. Sacca, R. Meyer, C.M. Niemeyer, Temperature-dependent FRET spectroscopy for the high-throughput analysis of self-assembled DNA nanostructures in real time, Nat. Protoc. 4 (3) (2009) 271.

[47] M. Benelmekki, S. Gasso, L.M. Martinez, Simultaneous optical and magnetophoretic monitoring of DNA hybridization using superparamagnetic and plasmonic colloids, Colloids Surf. B 193 (2020) 111126.

[48] J. Storhoff, A. Lazarides, R. Mucic, C. Mirkin, R. Letsinger, G. Schatz, What controls the optical properties of DNA-linked gold nanoparticle assemblies? J. Am. Chem. Soc. 122 (19) (2000) 4640-4650.

[49] N. Leopold, B. Lendl, A new method for fast preparation of highly surface-enhanced Raman scattering (SERS) active silver colloids at room temperature by reduction of silver nitrate with hydroxylamine hydrochloride, J. Phys. Chem. B 107 (24) (2003) 5723-5727.

[50] E. Papadopoulou, S.E. Bell, Label-free detection of single-base mismatches in DNA by surface-enhanced Raman spectroscopy, Angew. Chem. Int. Ed. 50 (39) (2011) 9058-9061. 
[51] A. Stewart, S. Zheng, M.R. McCourt, S.E. Bell, Controlling assembly of mixed thiol monolayers on silver nanoparticles to tune their surface properties, ACS Nano 6 (5) (2012) 3718-3726.

[52] B. Ha, K. Char, Conformational behavior of dodecyldiamine inside the confined space of montmorillonites, Langmuir 21 (18) (2005) 8471-8477.

[53] G. D’Acunto, F. Ripanti, P. Postorino, M.G. Betti, M. Scardamaglia, C. Bittencourt, C. Mariani, Channelling and induced defects at ion-bombarded aligned multiwall carbon nanotubes, Carbon 139 (2018) 768-775.

[54] N.J. Halas, S. Lal, W.-S. Chang, S. Link, P. Nordlander, Plasmons in strongly coupled metallic nanostructures, Chem. Rev. 111 (6) (2011) 3913-3961.

[55] F. Brasili, A. Capocefalo, D. Palmieri, F. Capitani, E. Chiessi, G. Paradossi, F. Bordi, F. Domenici, Assembling patchy plasmonic nanoparticles with aggregationdependent antibacterial activity, J. Colloid Interface Sci. 580 (2020) 419-428.

[56] H. Deng, V.A. Bloomfield, J.M. Benevides, G.J. Thomas Jr., Dependence of the Raman signature of genomic B-DNA on nucleotide base sequence, Biopolymers 50 (6) (1999) 656-666.

[57] L. Movileanu, J. M. Benevides, G. J. Thomas Jr, Temperature dependence of the raman spectrum of DNA. II. Raman signatures of premelting and melting transitions of poly (dA). poly (dT) and comparison with poly (dA-dT). poly (dA-dT), Biopolymers 63 (3) (2002) 181-194.

[58] D. Caprara, F. Ripanti, A. Capocefalo, A. Sarra, F. Brasili, C. Petrillo, C. Fasolato, P. Postorino, DNA-functionalized gold nanoparticle assemblies for surface enhanced Raman scattering, Colloid Surf. A: Physicochem. Eng. Asp. 589 (2020) 124399.

[59] F.J. Colas, M. Cottat, R. Gillibert, N. Guillot, N. Djaker, N. Lidgi-Guigui, T. Toury, D. Barchiesi, A. Toma, E. Di Fabrizio, P.G. Gucciardi, M.L. de la Chapelle, Red-shift effects in surface enhanced Raman spectroscopy: spectral or intensity dependence of the near-field? J. Phys. Chem. C 120 (25) (2016) 13675-13683.

[60] L.A. Gearheart, H.J. Ploehn, C.J. Murphy, Oligonucleotide adsorption to gold nanoparticles: a surface-enhanced Raman spectroscopy study of intrinsically bent DNA, J. Phys. Chem. B 105 (50) (2001) 12609-12615.

[61] J.N. Zadeh, C.D. Steenberg, J.S. Bois, B.R. Wolfe, M.B. Pierce, A.R. Khan, R.M. Dirks, N.A. Pierce, NUPACK: analysis and design of nucleic acid systems, J. Comput. Chem. 32 (1) (2011) 170-173.

[62] C. H. Choi, G. Kalosakas, K. Rasmussen, M. Hiromura, A. R. Bishop, A. Usheva, DNA dynamically directs its own transcription initiation, Nucleic Acids Res. 32 (4) (2004) 1584-1590.

[63] T.S. van Erp, S. Cuesta-Lopez, J.-G. Hagmann, M. Peyrard, Can one predict DNA transcription start sites by studying bubbles? Phys. Rev. Lett. 95 (21) (2005) 218104.

[64] D.A. Braasch, D.R. Corey, Locked nucleic acid (LNA): fine-tuning the recognition of DNA and RNA, Chem. Biol. 8 (1) (2001) 1-7.

[65] G.W. Hatfield, C.J. Benham, DNA topology-mediated control of global gene expression in Escherichia coli, Annu. Rev. Genet. 36 (1) (2002) 175-203.

[66] S. Mahajan, J. Richardson, T. Brown, P.N. Bartlett, SERS-melting: a new method for discriminating mutations in DNA sequences, J. Am. Chem. Soc. 130 (46) (2008) 15589-15601.

[67] D. Macdonald, E. Smith, K. Faulds, D. Graham, DNAw detection by SERS: hybridisation parameters and the potential for asymmetric PCR, Analyst 145 (5) (2020) 1871-1877.

[68] F. Liu, E. Tøstesen, J.K. Sundet, T.-K. Jenssen, C. Bock, G.I. Jerstad, W.G. Thilly, E. Hovig, The human genomic melting map, PLoS Comput. Biol. 3 (5) (2007), e93. 